\title{
Body reactions during chain saw work
}

\author{
T MATOBA, M CHIBA, AND T SAKURAI \\ From the Department of Environmental Medicine, Kurume University School of Medicine, Kurume, Japan
}

ABSTRACT Body reactions during chain saw work were studied in 14 subjects. The subjects divided into three groups (control, sulpiride, and propranolol) successively cut down logs with a chain saw for seven minutes. The start of the sawing led to a pronounced increase in heart rate which persisted during the sawing. The groups taking sulpiride and propranolol showed a smaller increase in heart rate compared with the controls. Hormonal values (adrenocorticotropic hormone (ACTH), cortisol, adrenaline (Ad), noradrenaline (NA), and dopamine) were increased by the operation. A comparison of these values before and after the operation showed that the increase of cortisol, Ad, and NA values was highest in the controls, intermediate in the propranolol group, and lowest in the sulpiride group. The increase in ACTH, however, was greatest in the sulpiride group, intermediate in the controls and correct in the propranolol group. These findings provide some evidence that chain saw work may have an influence on the whole body, including the hypothalamus and the limbic lobe of the brain.

The habitual use of vibrating tools such as chain saws, pneumatic hammers, or electric hammers has resulted in injury to the peripheral blood vessels and nerves, giving rise to Raynaud's phenomenon in the fingers. ${ }^{1}$ The patients, however, are afflicted not only with Raynaud's phenomenon and numbness in the fingers but insomnia, palmar hyperhydrosis, headache, fatiguability, tinnitus, and loss of libido. ${ }^{1}$ These findings suggest that there may be disorders of the central as well as the autonomic nervous system. Indeed, abnormalities in electroencephalograms and optokinetic nystagmus may be elicited by vibration and noise. ${ }^{1-3}$ The major factors affecting the human body are vibration and noise generated from vibrating tools, a cold environment, and emotional factors. ${ }^{4}$ These stressors stimulate the body, leading to various symptoms and signs, some of which may be an adaptive response to the stressors, a defence mechanism. ${ }^{5}$ This paper presents some physiological and hormonal data to indicate the ways in which the body reacts to chain saw work.

\section{Subjects and methods}

The subjects were 14 men who had worked as chain saw operators for, on average, 14 years; their mean age was $44 \pm 8$ (mean $\pm S D$ ). All subjects underwent physical and laboratory examinations to exclude the

Received 7 January 1985

Accepted II February 1985 possibility of vibration disease and cardiovascular diseases. None complained of Raynaud's phenomenon in the fingers, but some had slight numbness in the fingers and arms, palmar hyperhydrosis, or shoulder stiffness. The cumulative operation time was from 0920 to 1690 .

The subjects were divided into three groups: group 1 consisted of six control subjects, group 2 of four men with intramuscular premedication of $100 \mathrm{mg}$ sulpiride, one of the benzamide derivatives, and group 3 of four men who received $20 \mathrm{mg}$ propranolol administered by mouth. No significant differences were noted between these three groups.

As shown in fig 1 , the experimental procedures are as follows. Firstly, after explaining the experimental purposes and procedures to all the subjects, laboratory examinations were performed: electrocardiograms (12 limb and precordial leads), blood pressure, digital plethysmography with auditory stimuli for detecting the sympathetic nervous tones, ${ }^{67}$ and blood sampling. Blood chemistry included estimations of adrenocorticotropic hormone (ACTH), cortisol, adrenaline, noradrenaline, and dopamine concentrations. A second examination was then made after the administration of sulpiride or propranolol. After these procedures, the subjects operated chain saws for seven minutes. Electrocardiographic recordings with a bipolar lead on the chest and the electroencephalographic recordings with two monopolar leads from left and right frontal poles were made during the successive cutting of logs with a chain saw. 
The chain saws used were McCulloch PM850 $(82 \mathrm{ml}$ of engine capacity, $10 \mathrm{~kg}$ in weight, $22 \mathrm{~m} / \mathrm{s}^{2}$ of vibration level, and $109.6 \mathrm{~dB}(\mathrm{~A})$ of noise level) or Husqvarna $285 C D(85 \mathrm{ml}$ of engine capacity, $11 \mathrm{~kg}$ in weight, $23 \mathrm{~m} / \mathrm{s}^{2}$ of vibration level, and $103.4 \mathrm{~dB}(\mathrm{~A})$ of noise level).

ACTH and cortisol were analysed by the radioimmunoassay (RIA) method, and catecholamines (adrenaline, noradrenaline, and dopamine) were measured by high performance liquid chromatography (tridehydro-oxyindol method).

Smoking was prohibited during the study. The outdoor temperature was $20-23^{\circ} \mathrm{C}$ and the humidity $55 \%$. The procedure took four hours and started at 0900. Statistical significances were determined by Student's paired or unpaired $t$ test as appropriate.

\section{Results}

\section{HEART RATES}

Changes in heart rate were measured before, during, and after operating the chain saw. The heart rate was changed by supine, sitting, or standing postures. The change from a supine to a sitting posture led to an increase in heart rate in the controls. By contrast, the heart rates in the sulpiride and propranolol groups decreased (fig 2). Change from the sitting to the standing position, however, produced a significant increase in the heart rate in all groups.

The start of sawing produced a pronounced, statistically significant increase in heart rate $(p<0.005$ and $p<0.05)$. The increase in heart rate persisted during

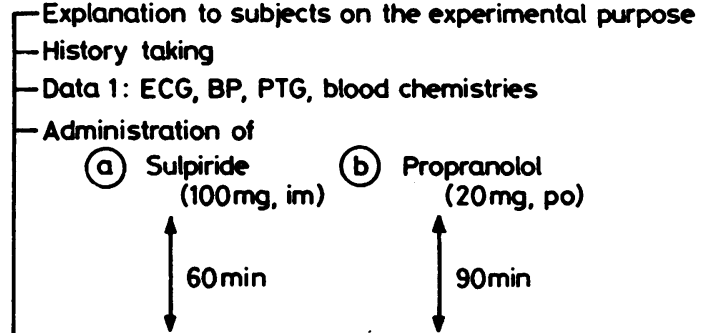

-Data 2: BP, PTG, blood chemistries

-Operating a chain saw for $7 \mathrm{~min}$

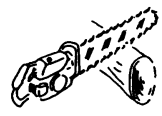

Data 3: BP, PTG, blood chemistries

\section{No smoking during experiments}

Fig 1 Protocol of experimental procedures.

the sawing: the average heart rates in the control, sulpiride, and propranolol groups were 101, 92, and 75 beats/min, respectively. After finishing the operation, the increased heart rates in the sulpiride and propranolol groups rapidly reverted to the preoperational level, but in the controls the decrease was less pronounced. The chain saw operation may stimulate the sympathetic nervous system, resulting in an increase in heart rate. The control of heart rate is centrally modulated by an interaction between the hypothalamus and the medulla. ${ }^{8}$ The change in the heart rates in the sulpiride and propranolol groups would be due to the suppression of the hypothalamus by sulpiride and the blocking of beta-receptor sites by propranolol.

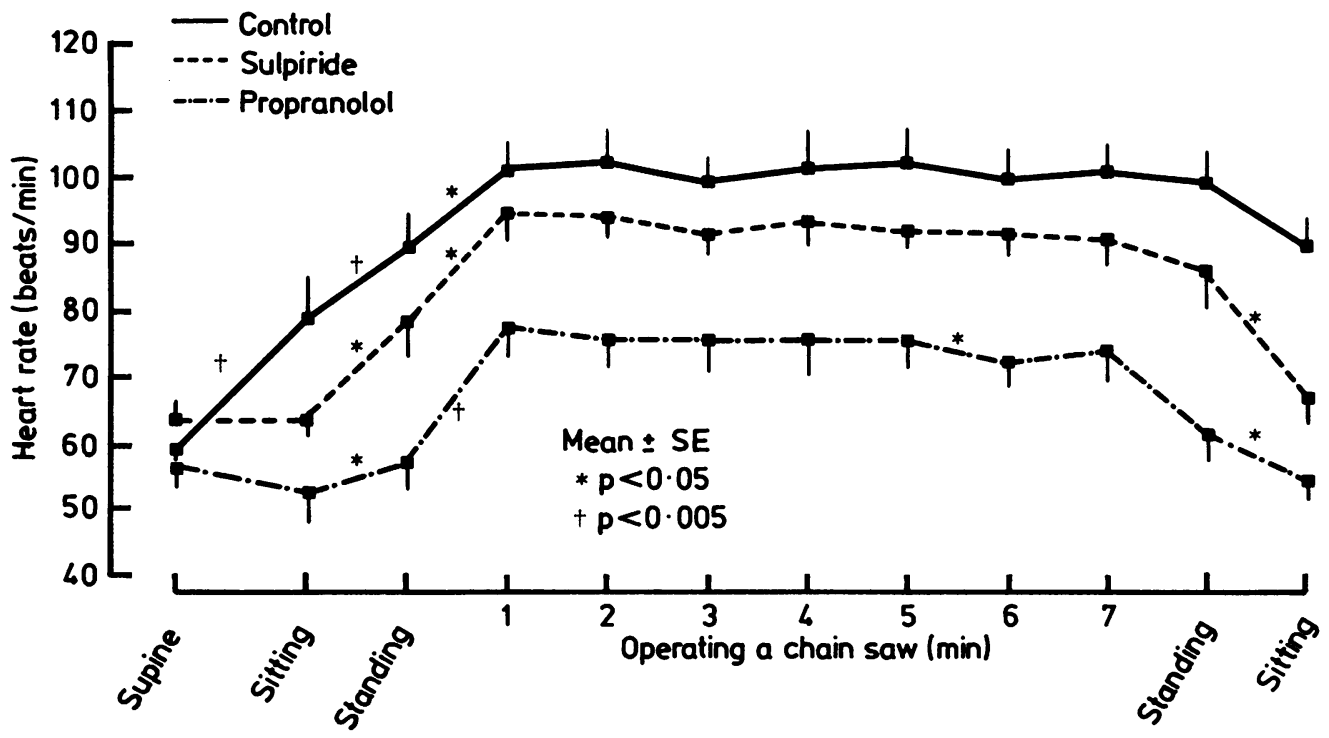

Fig 2 Change in heart rate before, during, and after chain saw work. 


\section{ELECTROCARDIOGRAMS}

The resting electrocardiographic (ECG) findings were within normal limits in all subjects. In the controls the amplitude of the $\mathbf{R}$ waves in the ECG was decreased by approximately $15 \%$ of the original value during the chain saw operation. This may be partly explained by Brody's effects. ${ }^{9}$ By contrast, the amplitude of the $\mathbf{R}$ waves was increased in the sulpiride and propranolol groups, although the increase was within $10 \%$. After finishing the operation, the amplitude of the $R$ waves recovered to the original values within one minute.

The ratio of $T$ to $R$ deflections in the control group increased during sawing whereas in the sulpiride and propranolol groups the $\mathbf{T} / \mathbf{R}$ ratios decreased to approximately $80 \%$ to $90 \%$ of the original level. No statistically significant differences were observed between the groups.

\section{ELECTROENCEPHA LOGRAMS}

The electroencephalographic (EEG) findings showed that beta activity was predominant in the controls and the propranolol group during sawing. In the sulpiride group beta and alpha activities appeared almost equally. These findings may be accounted for by a partial suppression of beta activity by sulpiride in the brain.

\section{DIGITAL PLETHYSMOGRAMS}

In the controls the amplitudes of the digital plethysmogram before and after the sawing were $5.4 \pm 0.34$ (mean $\pm \mathrm{SE}$ ) and $6.3 \pm 0.92 \mathrm{mV} / \mathrm{V}$, respectively. Sulpiride significantly increased the amplitude from $3.5 \pm 0.92$ to $6.3 \pm 2.15 \mathrm{mV} / \mathrm{V}(\mathrm{p}<0.05)$. This suggests that the hypertonicity of the sympathetic nerve is relieved by sulpiride. In the digital plethysmography with auditory stimuli ${ }^{67}$ the sympathetic hyperreactive types were changed to the normoreactive type by the administration of sulpiride. By contrast, the effect of propranolol on the amplitude was scarcely detectable.

\section{BLOOD PRESSURES}

No appreciable changes in blood pressure were observed before and after the chain saw operation in any of the groups.

The product of systolic blood pressure and heart rate was significantly reduced by propranolol $(\mathrm{p}<$ 0.01 ) but was scarcely affected by sulpiride.

\section{HORMONAL CHANGES}

ACTH, cortisol, adrenaline noradrenaline, and dopamine concentrations in the plasma were measured before and after the chain saw operation. In all subjects the values of dopamine were less than $200 \mathrm{pg} / \mathrm{ml}$, and there were negligible changes.

All hormonal values in the controls were increased by the operation (fig 3) but were statistically significant only for plasma cortisol $(p<0.01)$. Administration of sulpiride led to a statistically significant decrease in the values of ACTH, cortisol, and noradrenaline $(p<0.05)$ but adrenaline increased only slightly. Under the influence of propranolol, no appreciable changes were observed in the values of ACTH, cortisol, and adrenaline, although noradrenaline was moderately decreased.

The chain saw operation resulted in an increase in all hormones. A comparison of these values before and after the operation showed that the increases of cortisol, adrenaline, and noradrenaline concentrations were highest in the controls, intermediate in the propranolol group, and lowest in the sulpiride group (fig 4). The increase in ACTH, however, showed a different pattern, being greatest in the sulpiride group, intermediate in the controls, and lowest in the propranolol group. Thus the rates of increase induced by the chain saw operation were smallest in the subjects pretreated with sulpiride. These findings may be interpreted as showing that sulpiride suppresses the hypothalamic excitation induced by the chain saw operation. In other words, chain saw work may have an influence on the whole body, including the hypothalamus and the limbic lobe in the brain.

\section{Discussion}

During chain saw work, the human body is exposed to three major stressors: vibration, noise, and cold. ${ }^{4}$ In general, the vibration level of a chain saw is from 5.6 to $28 \mathrm{~m} / \mathrm{s}^{2}$, and the noise level is from 92 to 110 $\mathrm{dB}$ (air). These intensities of vibration and noise levels can actually stress the human body, leading to various symptoms and signs consisting not only of injuries to the peripheral circulatory, nervous, muscular, bone, and articular systems, but also of disorders of the autonomic nervous and central nervous systems including acoustic nerve impairment and optokinetic nystagmus. In the cardiovascular system an adaptive response to the stressors has been observed as a defence mechanism. ${ }^{5}$

In the present study chain saw work led to changes in the heart rate, in the electrocardiogram and electroencephalogram, and in plasma hormonal levels, although the duration of the work was only seven minutes. These results could be interpreted as showing that the reaction to chain saw work occurs in the whole body. It may also directly or indirectly stimulate the central nervous system. Pyykkö and coworkers showed that optokinetic nystagmus could be evoked by exposure to vibration and noise ${ }^{3}$ and clinically, patients with vibration disease may show symptoms and signs of disorders of thermoregulation, of the autonomic and central nervous systems, and of the 


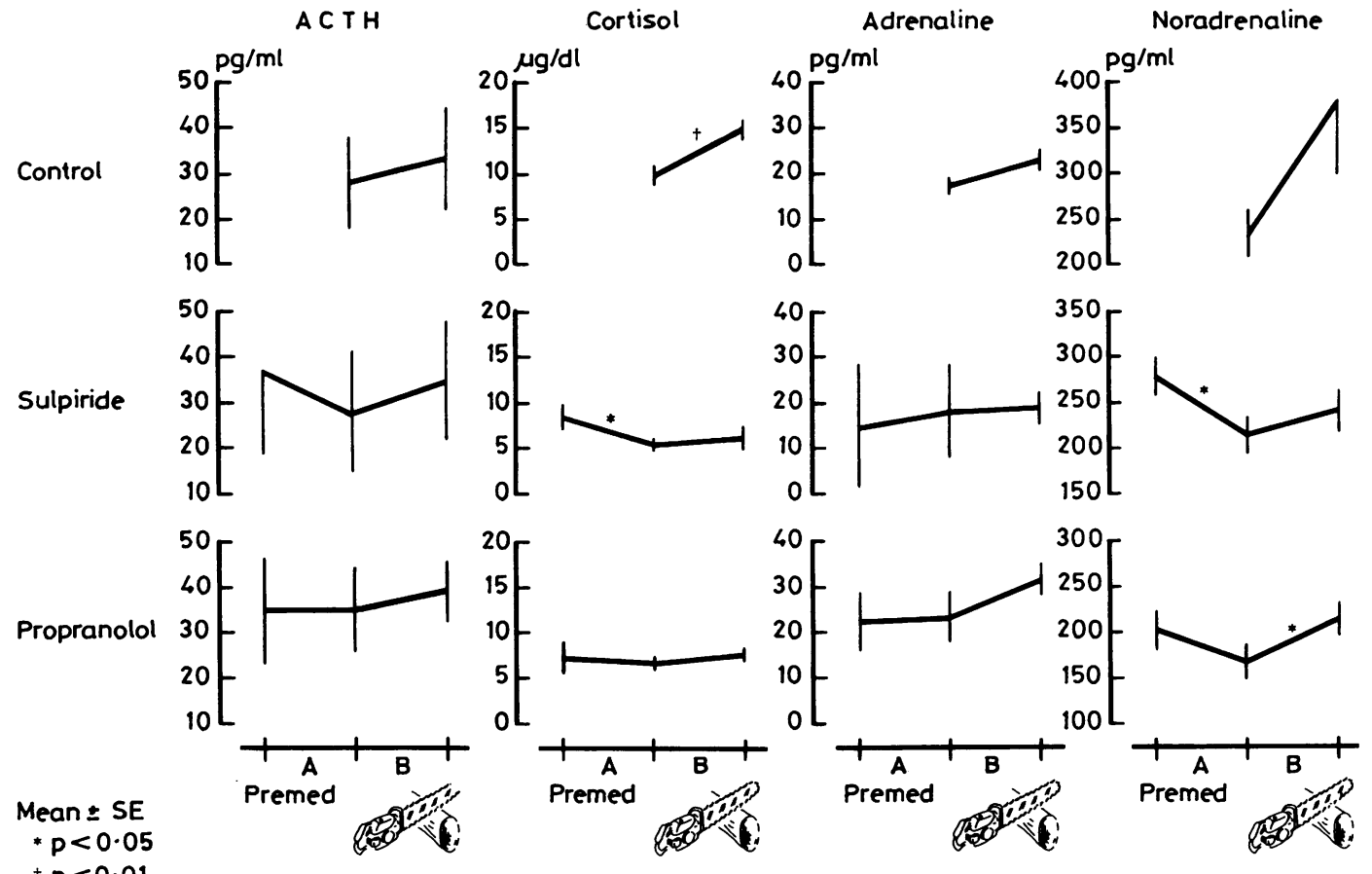

Fig 3 Changes in plasma hormone levels induced by chain saw work.

ACTH

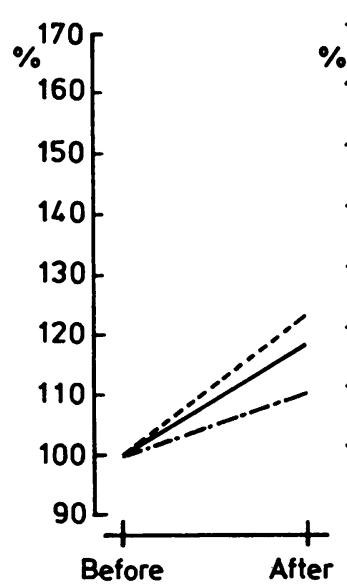

- Control

-...- Sulpiride

-... Propranolol

\section{Cortisol}

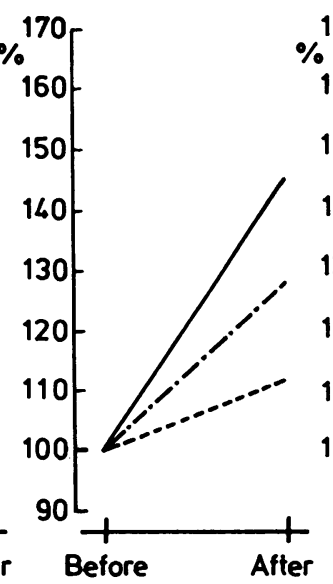

Adrenaline

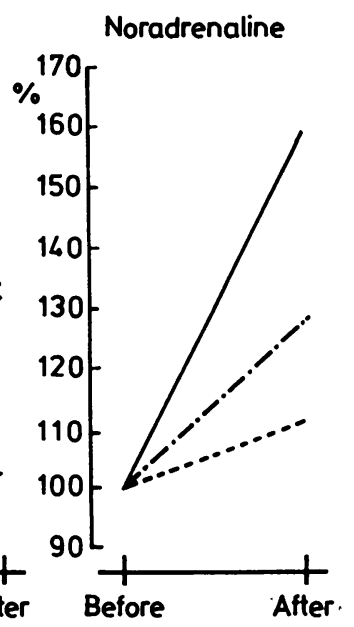

Fig 4 Percentage changes in plasma hormone levels before and after chain saw work.

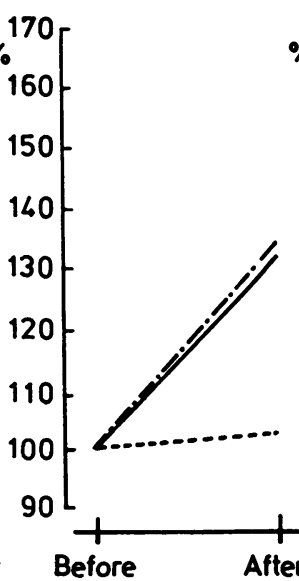


cardiovascular system. ${ }^{15}$ The responses of the body after premedication with sulpiride or propranolol differ, however. For instance, plasma hormonal levels are decreased by sulpiride, although scarcely at all by propranolol. The reason for this difference may be due to the pharmacokinetics of the drugs: sulpiride acts mainly on the limbic system, including the hypothalamus, and blocks the neurotransmission of dopamine. ${ }^{1011}$ On the other hand, propranolol acts directly on the target organs. ${ }^{12}$ These findings may provide evidence that chain saw work exerts a strong influence not only on the peripheral circulatory, nervous, motor, and support systems, but on the whole body of the operator.

This work was supported by a research grant for cardiovascular diseases $(56-1)$ from the Ministry of Health and Welfare in Japan.

\section{References}

${ }^{1}$ Matoba T, Kusumoto H, Mizuki Y, Kuwahara H, Inanaga K, Takamatsu $\mathrm{M}$. Clinical features and laboratory findings of vibration disease: a review of 300 cases. Tohoku J Exp Med 1977;123:57-65.

${ }^{2}$ Arikawa K, Shirakawa T, Kotorii T, et al. An electroencephalographic study of patients with vibration disease. Folia Psychiatr Neurol Jpn 1978;32:211-22.
${ }^{3}$ Pyykkö I, Månsson M, Matsuda I, Ito S, Hinoki M. Effects of pure-tone sound, impulse noise, and vibration on visual orientation. Am J Otopharyngol 1982;3:104-11.

${ }^{4}$ Matoba T, Chiba M, Toshima H. Cardiovascular features of the vibration syndrome: an adaptive response. In: Brammer AJ, Taylor W, eds. Vibration effects on the hand and arm in industry. London: John Wiley and Sons, 1983:25-30.

${ }^{5}$ Matoba T, Itaya M, Toyomasu K, Tsuiki T, Toshima H, Kuwahara $H$. Increased left ventricular functions as an adaptive response in vibration disease. Am J Cardiol 1983;51:1223-6.

${ }^{6}$ Matoba T, Kusumoto H, Omura H, Kotorii T, Kuwahara H, Takamatsu M. Digital plethysmographic responses to auditory stimuli in patients with vibration disease. Tohoku $J$ Exp Med 1975;115:385-92.

${ }^{7}$ Matoba T, Mizobuchi H, Ito H, Chiba M, Toshima H. Further observations of the digital plethysmography in response to auditory stimuli and its clinical applications. Angiology 1981; 32:62-72.

${ }^{8}$ Manning JW. Central nervous system control of cardiac rhythm. Cardiology 1976;61:7-19.

${ }^{9}$ Brody AB. A theoretical analysis of intracavitary blood mass influence on the heart-lead relationship. Circ Res 1956;4:731-8.

${ }^{10}$ Yoshida M. The effect of psychotrophic drug, sulpiride, on the catecholaminergic systems in rats: behavioral and fluorescence histochemical studies. Japanese Journal of Neuropsychopharmacology 1981;3:45-55.

${ }^{11}$ Baldessarini RJ. Drugs and the treatment of psychiatric disorders. In: Goodman Gilman A, Goodman LS, Gilman A, eds. The pharmacological basis of therapeutics. New York: MacMillan Publishing Co Inc, 1980:391-447.

12 Weiner N. Drugs that inhibit adrenergic nerves and block adrenergic receptors. In: Goodman Gilman A, Goodman LS, Gilman A, eds. The pharmacological basis of therapeutics. New York: MacMillan Publishing Co Inc 1980:176-210. 